\title{
Step-Response Model Development for Dynamic Matrix Control of a Drum-Type Boiler-Turbine System
}

\author{
Un-Chul Moon and Kwang. Y. Lee, Life Fellow, IEEE
}

\begin{abstract}
This paper presents the application of dynamic matrix control (DMC) to a drum-type boiler-turbine system. Two types of step-response models for DMC are investigated in designing the DMC; one is developed with the linearization of the nonlinear plant model and the other is developed with the process step-response data. Then, the control performances of the DMC based on both types of models are evaluated. Because of severe nonlinearity of drum water-level dynamics, it is observed that the simulation with the step-response model based on the test data shows satisfactory results, while the linearized model is not suitable for controller design of the drum-type boiler-turbine system.
\end{abstract}

Index Terms-Boiler-turbine control, dynamic matrix control (DMC), power generation control, predictive control, process control.

\section{INTRODUCTION}

A BOILER-TURBINE system provides high-pressure steam to drive the turbine in thermal electric power generation. The purpose of the boiler-turbine system control is to meet the load demand of electric power while maintaining the pressure and water level in the drum within tolerance. This boiler-turbine system is usually modeled with a multi-inputmulti-output (MIMO) nonlinear system [1].

The severe nonlinearity and wide operation range of the boiler-turbine plant have resulted in many challenges to power system control engineers. Hogg and Ei-Rabaie presented an application of adaptive control, that is, the self-tuning generalized predictive control to a boiler system [2]. Cori and Maffezzoni applied a linear quadratic Gaussian (LQG) controller [3], Pellegrinetti and Bentsman designed an $H_{\infty}$ controller for boilers [4], Ben-Abdennour and Lee applied the LQG with loop transfer recovery (LQG/LTR) method [5], and Tan and others approximated the $H_{\infty}$ to PI controller [6]. These controllers are designed using the mathematical model of the underlying plant. Apart from these model-based controller designs, many kinds

Manuscript received October 15, 2008. Current version published May 19, 2009. This work was supported in part by the Electrical Engineering and Science Research Institute (EESRI), which is funded by the Ministry of Commerce, Industry and Energy, Korea, under Grant R-2005-B-103 and in part by the Manpower Development Program for Energy and Resources, which is funded by the Ministry of Knowledge and Economy (MKE). Paper no. TEC-00066-2007.

U. C. Moon is with the School of Electrical and Electronics Engineering, Chung-Ang University, Seoul 156-756, Korea (e-mail: ucmoon@cau.ac.kr).

K. Y. Lee is with the Department of Electrical and Computer Engineering, Baylor University, Waco, TX 76798-7356 USA (e-mail: kwang_y_lee@baylor.edu).

Digital Object Identifier 10.1109/TEC.2009.2015986 of artificial intelligence techniques have also been applied for nonmodel-based controller design [7]-[10].

Model predictive control (MPC) refers to a class of control algorithms that compute a sequence of control inputs based on an explicit prediction of outputs within some future horizon. The computed control inputs are typically implemented in a receding horizon fashion, meaning only the inputs for the current time are implemented and the whole calculation is repeated at the next sampling time [11], [12]. Therefore, one of the most important strengths of MPC is that it can consider the constraints of input and output variables that often exist in real industrial systems. Now, MPC has become a standard tool for process controls [12].

One of the most well-known MPC algorithms for the process control is dynamic matrix control (DMC), which assumes a step-response model for the underlying system. The multivariable DMC controller has been discussed extensively in the past [13]-[19]. Because DMC needs much numerical calculation at every sampling time, it is a suitable technique for the systems with slow dynamics. DMC has been successfully applied to numerous industrial processes, and many commercial softwares have been developed: DMC +, SMC, RMPCT, HIECON, PFC, $\mathrm{OPC}$, etc.

Nowadays, the MPC based on the state-space model has been developed [20]-[22]. However, it is hard to find industrial applications of the MPC based on state-space models. Prasad and others [23] presented simulation results of nonlinear physical MPC to the drum-type boiler-turbine system with disturbance and Prasad [24] proposed a nonlinear physical MPC based on principal component analysis to the drum-type boiler-turbine system in the case of sensor malfunction. In these papers, they used continuous linearization of a nonlinear physical model. In spite of these advanced developments of nonlinear MPC algorithm, the use of a single model at one operating point is standard practice in many industrial applications [25], [26].

Rovnak and Corlis [27] discussed theoretical and practical aspects of DMC, and presented simulation results of a supercritical boiler. In their paper, DMC was applied to supercritical boiler that has three outputs: electric power, steam pressure, and steam temperature. In the drum-type boiler-turbine system, however, drum water-level control is considered to be a more difficult problem, main reason being a nonminimum phase behavior due to shrink/swell and instability because of the integrating properties of drum-level dynamics [28]. Although many successful applications of DMC to various industrial plants have been reported, it is difficult to find the application of DMC to drum-type boiler-turbine system. Sanchez and others [29] 
presented an application of DMC to steam temperature control of fossil power plants, and showed that the single-input-singleoutput (SISO) DMC performs better than the PID control.

In this paper, we present a comparative study of DMC to a drum-type boiler-turbine system in a fossil power plant. In a practical design of a DMC, one of the most important steps is the development of a step-response model to describe the system dynamics. Here, two types of methods are considered for the development of the step-response model. One is when the exact nonlinear mathematical model is given, the step-response model can be found by linearizing the nonlinear model, and the other is when the step-response model is identified from the process test data. A DMC is designed for each model, and the control performances are evaluated and discussed. In addition, this paper demonstrates the advantages of using process step-response data over linearizing a mathematical model, which might not be available or too complex for a practical large-scale real plant.

\section{BOILER-Turbine System Model}

\section{A. Nonlinear Boiler-Turbine System Model}

The model of Bell and Åström [1] is assumed as a real plant among various nonlinear models for the boiler-turbine system. The model represents a $160 \mathrm{MW}$ oil-fired drum-type boiler-turbine generator for overall wide-range simulations and is described by a third-order MIMO nonlinear state equation as follows [1]:

$$
\begin{aligned}
& \dot{x}_{1}=-0.0018 u_{2} x_{1}^{9 / 8}+0.9 u_{1}-0.15 u_{3} \\
& \dot{x}_{2}=\frac{\left[\left(0.73 u_{2}-0.16\right) x_{1}^{9 / 8}-x_{2}\right]}{10} \\
& \dot{x}_{3}=\frac{\left[141 u_{3}-\left(1.1 u_{2}-0.19\right) x_{1}\right]}{85} \\
& y_{1}=x_{1} \\
& y_{2}=x_{2} \\
& y_{3}=0.05\left(0.13073 x_{3}+100 a_{c s}+\frac{q_{e}}{9}-67.975\right)
\end{aligned}
$$

where

$$
\begin{aligned}
\alpha_{c s} & =\frac{\left(1-0.001538 x_{3}\right)\left(0.8 x_{1}-25.6\right)}{x_{3}\left(1.0394-0.0012304 x_{1}\right)} \\
q_{e} & =\left(0.854 u_{2}-0.147\right) x_{1}+45.59 u_{1}-2.514 u_{3}-2.096 .
\end{aligned}
$$

The three state variables $x_{1}, x_{2}$, and $x_{3}$ are drum steam pressure $\left(P\right.$ in $\left.\mathrm{kg} / \mathrm{cm}^{2}\right)$, electric power ( $E$ in megawatt), and steamwater fluid density in the drum $\left(\rho_{f}\right.$ in $\left.\mathrm{kg} / \mathrm{m}^{2}\right)$, respectively. The three outputs $y_{1}, y_{2}$, and $y_{3}$ are drum steam pressure $\left(x_{1}\right)$, electric power $\left(x_{2}\right)$, and drum water-level deviation ( $L$ in meters), respectively. The $y_{3}$, drum water-level $L$, is calculated using two algebraic calculations $\alpha_{c s}$ and $q_{e}$ that are the steam quality (mass ratio) and the evaporation rate (kilograms per second), respectively.

The three inputs $u_{1}, u_{2}$, and $u_{3}$ are normalized positions of valve actuators that control the mass flow rates of fuel, steam to the turbine, and feed water to the drum, respectively. Positions of valve actuators are constrained to $[0,1]$, and their rates of change per second are limited to

$$
\begin{aligned}
& -0.007 \leq \frac{d u_{1}}{d t} \leq 0.007 \\
& -2.0 \leq \frac{d u_{2}}{d t} \leq 0.02 \\
& -0.05 \leq \frac{d u_{3}}{d t} \leq 0.05
\end{aligned}
$$

\section{B. Step-Response Model With Linearization}

In most cases of designing boiler-turbine control systems, it is assumed that the exact mathematical model is given, therefore, the linearization of the nonlinear mathematical model is used to design the linear controller [3]-[6]. With this assumption, the step-response model for DMC can be developed with the familiar linearization technique.

In using the nonlinear model, (1)-(8) is linearized using Taylor series expansion at the operating point, $\bar{y}_{0}=$ $\left(y_{10}, y_{20}, y_{30}\right), \bar{x}_{0}=\left(x_{10}, x_{20}, x_{30}\right), \bar{u}_{0}=\left(u_{10}, u_{20}, u_{30}\right)$.

The result of linearization is as follows:

$$
\begin{aligned}
& \dot{\bar{x}}=A \bar{x}(t)+B \bar{u}(t) \\
& \bar{y}(t)=C \bar{x}(t)+D \bar{u}(t)
\end{aligned}
$$

where

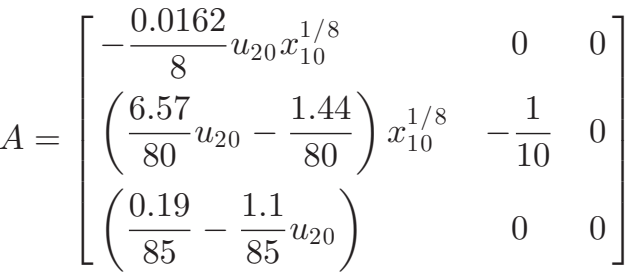

$$
\begin{aligned}
& B=\left[\begin{array}{ccc}
0.9 & -0.0018 x_{10}^{9 / 8} & -0.15 \\
0 & \frac{0.73}{10} x_{10}^{9 / 8} & 0 \\
0 & -\frac{1.1}{85} x_{10} & \frac{141}{85}
\end{array}\right]
\end{aligned}
$$

$C=\left[\begin{array}{ccc}1 & 0 & 0 \\ 0 & 1 & 0 \\ \left(5 \frac{\partial}{\partial x_{1}} a_{c s}+\frac{0.05}{9} \frac{\partial}{\partial x_{1}} q_{e}\right) & 0 & \left(0.065+5 \frac{\partial}{\partial x_{3}} a_{c s}\right)\end{array}\right]$

$$
D=\left[\begin{array}{ccc}
0 & 0 & 0 \\
0 & 0 & 0 \\
0.2533 & 0.00474 x_{10} & -0.014
\end{array}\right]
$$

The variables $\bar{y}, \bar{x}$, and $\bar{u}$ are the differences of the output, state, and input, respectively, from the corresponding operating points.

The operating points are determined based on a nominal operation of the plant. Considering that the model represents a $160 \mathrm{MW}$ unit, the operating point for power output $y_{2}$ is assumed as $85 \mathrm{MW}$, which is around its midpoint. This yields the corresponding pressure $y_{1}$ to be $115 \mathrm{~kg} / \mathrm{cm}^{2}$ from the 
balance of the plant model. The operating point for water level $y_{3}$ is zero in order to keep the water level in the middle of the drum. Then, the operating points for other remaining variables can be calculated by neglecting the derivative terms in (1)(9). The resulting operating points are $\bar{y}_{0}=(115,85,0), \bar{x}_{0}=$ $(115,85,402.759), \bar{u}_{0}=(0.4147,0.7787,0.5436)$.

The constant matrices $A, B, C$, and $D$ are evaluated at these operating points as follows:

$$
\begin{aligned}
A & =\left[\begin{array}{ccc}
-0.002854 & 0 & 0 \\
0.083152 & -0.1 & 0 \\
-0.007842 & 0 & 0
\end{array}\right] \\
B & =\left[\begin{array}{ccc}
0.9 & -0.374591 & -0.15 \\
0 & 15.191754 & 0 \\
0 & -0.148126 & 1.658824
\end{array}\right] \\
C & =\left[\begin{array}{ccc}
1 & 0 & 0 \\
0 & 1 & 0 \\
0.007566 & 0 & 0.004257
\end{array}\right] \\
D & =\left[\begin{array}{ccc}
0 & 0 & 0 \\
0 & 0 & 0 \\
0.2533 & 0.543056 & -0.014
\end{array}\right]
\end{aligned}
$$

Then, a simple algebraic operation with Laplace transform gives transfer functions as follows:

$$
\begin{aligned}
Y(s) & =\left[C(s I-A)^{-1} B+D\right] U(s) \\
& =\left(\begin{array}{lll}
G_{11} & G_{12} & G_{13} \\
G_{21} & G_{22} & G_{23} \\
G_{31} & G_{32} & G_{33}
\end{array}\right) U(s)
\end{aligned}
$$

where the transfer functions $G_{i j}$ are determined as follows:

$$
\begin{aligned}
G_{11} & =\frac{0.9 s+0.09}{s^{2}+0.103 s+0.00028} \\
G_{12} & =\frac{-0.37 s-0.037}{s^{2}+0.103 s+0.00028} \\
G_{13} & =\frac{-0.15 s-0.015}{s^{2}+0.103 s+0.00028} \\
G_{21} & =\frac{0.0748}{s^{2}+0.103 s+0.00028} \\
G_{22} & =\frac{15.19 s+0.0122}{s^{2}+0.103 s+0.00028} \\
G_{23} & =\frac{-0.01247}{s^{2}+0.103 s+0.00028} \\
G_{31} & =\frac{0.2533 s^{3}+0.0328 s^{2}+0.0007 s-3.0 \times 10^{-6}}{s^{3}+0.103 s^{2}+0.00028 s} \\
G_{32} & =\frac{0.543 s^{3}+0.0524 s^{2}-0.00018 s+1.07 \times 10^{-6}}{s^{3}+0.102 s^{2}+0.00028 s} \\
G_{33} & =\frac{-0.014 s^{3}+0.0045 s^{2}+0.0006 s+2.5 \times 10^{-6}}{s^{3}+0.102 s^{2}+0.00028 s}
\end{aligned}
$$

With (24)-(32), the unit step-response model $\left\{s_{i j}\right\}$ is developed, where $s_{i j}$ represents the response $y_{i}$ of $G_{i j}$ with input $u_{j}$.

Fig. 1 shows the comparison of step responses with linearized step-response model (dotted line) and original non-
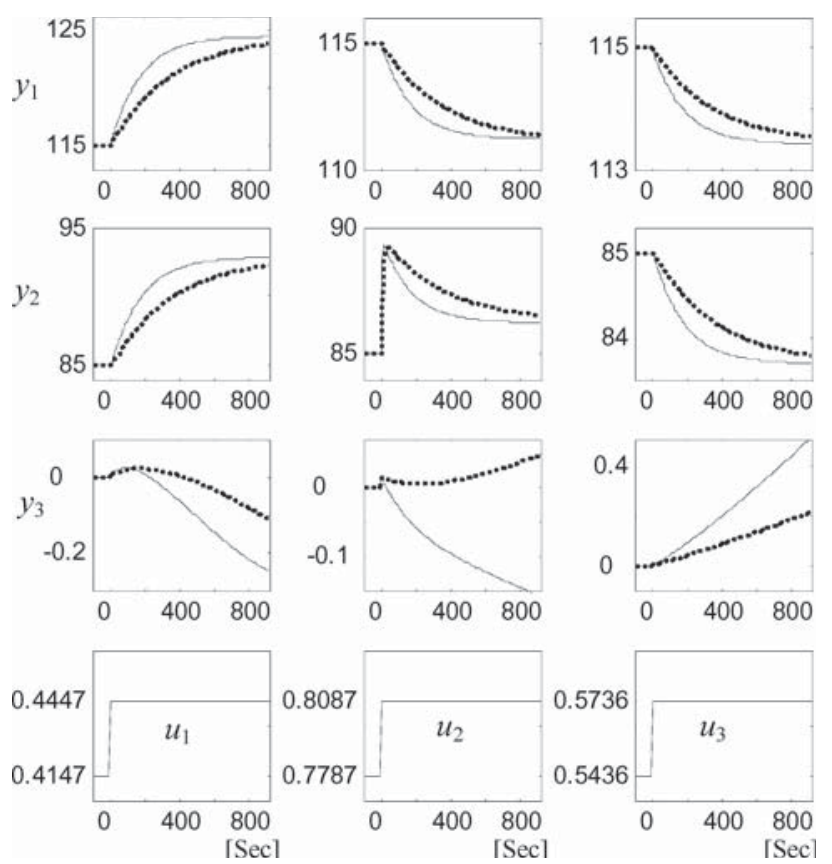

Fig. 1. Step-responses comparison of linearized model (dot) and nonlinear model (solid). Horizontal axes are time (in seconds), and the three rows of plots represent the outputs, $y_{1}\left(P\right.$ in $\left.\mathrm{kg} / \mathrm{cm}^{2}\right), y_{2}$ ( $E$ in megawatt), and $y_{3}(L$ in meters). The three columns of plots are the responses corresponding to the respective step inputs, $u_{1}, u_{2}$, and $u_{3}$.

linear model (solid line), when the system is at the operating points $\bar{y}_{0}=(115,85,0), \bar{x}_{0}=(115,85,402.759), \bar{u}_{0}=$ $(0.4147,0.7787,0.5436)$. In the figure, three inputs are independently applied as 0.03 , which is $3 \%$ of the entire range of valve position. Horizontal axes are time (in seconds), and vertical axes represent the outputs, $y_{1}\left(P\right.$ in $\left.\mathrm{kg} / \mathrm{cm}^{2}\right), y_{2}(E$ in megawatt), and $y_{3}$ ( $L$ in meters). The three columns of plots are the responses corresponding to the respective step inputs, $u_{1}, u_{2}$, and $u_{3}$.

We can observe the outputs $y_{1}$ and $y_{2}$ of linearized stepresponse model describe effectively the original nonlinear dynamics, while the output $y_{3}$ of linearized model shows some differences. The models $G_{31}$ and $G_{33}$ show some difference in slopes, but in the same direction. However, $G_{32}$, the response from the amount of governor steam to the drum water level, shows an opposite direction in the slope. Therefore, the quality of $G_{32}$ has a limitation to describe the original nonlinear dynamics. This point will be discussed in Section II-D.

\section{Step-Response Model With Process Test}

In the case when a reliable mathematical model is not available, the step-response model is obtained from experimental data. The usual process test signal is the step signal or a pseudorandom binary signal [25], [26].

In this paper, instead of the physical experiment a virtual experiment was performed to develop the step-response model by applying step inputs to the plant described by the nonlinear model (1)-(8). One of the important issues in the process test is the amplitude of the step. In general, large step input drives the 

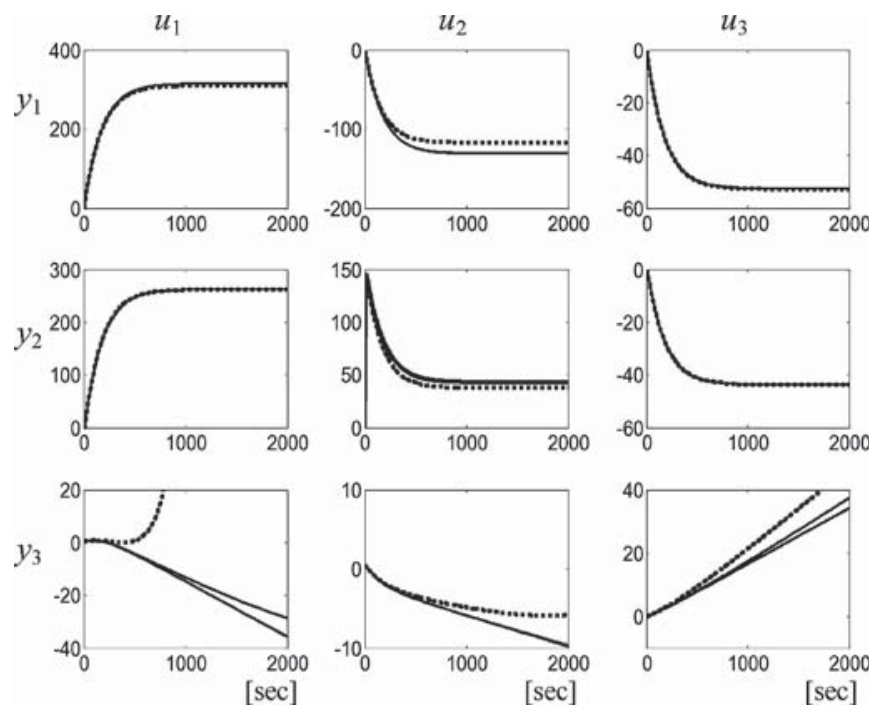

Fig. 2. Unit step-response models with different step inputs. Ten step inputs, $10^{-1}, 10^{-2}, \ldots, 10^{-10}$, are applied as inputs.

process output to nonlinear region, while the response of small step input is concealed in noise and disturbance signal. Therefore, an engineering judgment with compromise is necessary.

With the same operating point of the linearized model, $\bar{y}_{0}=$ $(115,85,0), \bar{x}_{0}=(115,85,402.759), \bar{u}_{0}=(0.4147,0.7787$, $0.5436)$, the three step inputs, $u_{1}, u_{2}$, and $u_{3}$, are applied independently, and then corresponding output responses are stored. To obtain the unit step response, output responses are normalized by dividing the outputs with the amplitude of the corresponding inputs.

To find out the effect of step input size, different step inputs are applied independently and corresponding unit stepresponse models are compared. Ten different step inputs, $10^{-1}$, $10^{-2}, \ldots, 10^{-10}$, are applied independently for $u_{1}, u_{2}$, and $u_{3}$. Fig. 2 shows the ten-unit step-response models, where the responses of $y_{1}$ and $y_{2}$ show little difference among the ten different responses. The responses of $y_{3}$ also show little difference except for the step size of $10^{-1}$. In the case when $u_{1}=0.1, y_{3}$ is diverged; therefore, the step input greater than 0.1 should be avoided.

In the Fig. 2, the unit step responses are almost the same when the inputs are smaller than $10^{-2}$. Therefore, the step size $10^{-2}$ is small enough to develop the step-response model to avoid the nonlinear region. Considering the noise and disturbance of the real process test, the step size of $10^{-2}$ is used in the process test in this paper. The step size $10^{-2}$ means the $1 \%$ of the entire range of valve position.

\section{Comparison of the Two Step-Response Models}

Fig. 3 shows a comparison of the two-unit step-response models, solid line is the unit step response from the process test with step size 0.01 and dotted line is the unit step response from the linearized model (24)-(32). In the DMC design, the stepresponse model in Fig. 3 is discretized with a sampling time. The discretized step-response coefficients are stored in a ma-
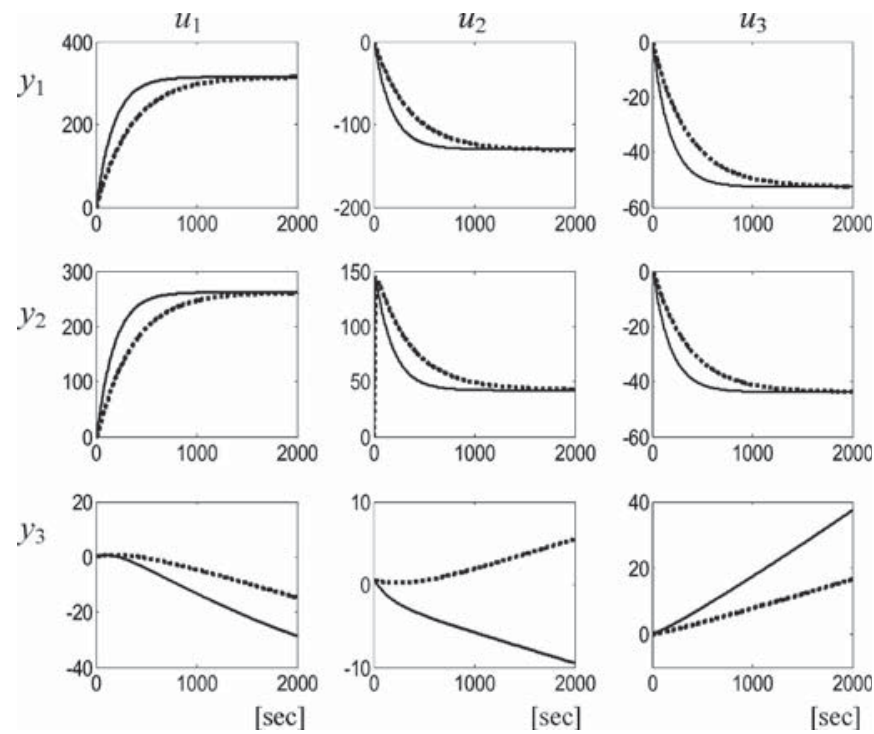

Fig. 3. A comparison between linearized model and process test model. Dotted line: by linearization; solid line: by process test.

trix form, called dynamic matrix, to describe the dynamics of nonlinear boiler-turbine system.

In Fig. 3, we can observe the notable difference of output $y_{3}$, drum level, as expected from Fig. 1. The models $G_{31}$ and $G_{33}$ show some difference in slopes, moreover, $G_{32}$, the response from the amount of governor steam to the drum water level, shows an opposite direction in the slope. Therefore, the $G_{32}$ obtained from the linearized model has a limitation. Considering that DMC use a long-range prediction of future output, poor performance is expected with linearized step-response model.

The linearization of a nonlinear system describes the dynamics with infinitesimal variable changes. Therefore, the linearized model is valid only in small variation of both input and output. The accuracy of the linearized model depends on the degree of nonlinearity. The model for the water level $y_{3}$ is highly nonlinear compared to that for variables $y_{1}$ and $y_{2}$ as shown in (1)-(8). Therefore, the linearized model for $y_{3}$ is not expected to be reliable. In fact, the linearized model for water level $y_{3}$ contains integration modes in (30)-(32), while $y_{1}$ and $y_{2}$ in (24)-(29) are stable in the bounded-input-bounded-output sense. As time increases, the water level $y_{3}$, due to the integral effect, keeps increasing or decreasing with the constant step input signal and finally goes out of the initial linearization region. Therefore, considering the fact that the DMC uses the step-response model that is obtained from a long-range prediction of future output, the performance with the linearized model has a limitation.

\section{DMC FOR BOILER-TURBINE SYSTEM}

The design of DMC in this paper follows the standard approach [12]. For a SISO system, the prediction equation is in the following form:

$$
Y_{k+1 \mid k}=Y_{k+1 \mid k-1}+S \Delta U_{k}+Y_{k+1 \mid k}^{d}
$$

where, $Y_{k+1 \mid k}$ is a $p \times 1$ vector representing a prediction of future output trajectory, $\left[y_{k+1 \mid k}, \ldots, y_{k+p \mid k}\right]^{T}$ at $t=k$, and $p$ is 
the prediction horizon; $Y_{k+1 \mid k-1}$ is a $p \times 1$ vector representing the unforced output trajectory $\left[y_{k+1 \mid k-1}, \ldots, y_{k+p \mid k-1}\right]^{T}$, which means the open-loop prediction while the input $u$ remains constant at the previous value $u_{k-1} ; \Delta U_{k}$ is an $m \times 1$ input adjustments vector $\left[\Delta u_{k}, \ldots, \Delta u_{k+m-1}\right]^{T}$ and $m$ is the control horizon; $Y_{k+1 \mid k}^{d}$ is a $p \times 1$ vector representing an estimate of unmeasured disturbance on the future output; and, $S$ is a $p \times m$ dynamic matrix containing the step-response coefficients as follows:

$$
S=\left[\begin{array}{cccc}
s_{1} & 0 & \cdots & 0 \\
s_{2} & s_{1} & \ddots & \vdots \\
s_{3} & s_{2} & \ddots & 0 \\
\vdots & \vdots & \ddots & s_{1} \\
\vdots & \vdots & \cdots & \vdots \\
s_{p} & s_{p-1} & \cdots & s_{p-m+1}
\end{array}\right]
$$

where $s_{i}$ is the amplitude of step response at the $i$ th sampling step.

To compute the inputs, the following online optimization is performed at every sampling time:

$$
\min _{\Delta U_{k}}\left\|E_{k+1 \mid k}\right\|_{\Lambda}+\left\|\Delta U_{k}\right\|_{\Gamma}
$$

where, $E_{k+1 \mid k}=Y_{k+1 \mid k}-R_{k+1 \mid k}=\left[e_{k+1}, \ldots, e_{k+p}\right]^{T}$ is a $p \times 1$ error vector, $R_{k+1 \mid k}=\left[r_{k+1}, \ldots, r_{k+p}\right]^{T}$ is a $p \times 1$ vector containing the desired trajectory of the future output, $\Lambda$ and $\Gamma$ are the weights for the weighted Euclidean norm of the corresponding vectors. To the above, the following additional constraints are added:

$$
\begin{aligned}
& Y_{\min } \leq Y_{k+1 \mid k} \leq Y_{\max } \\
& \Delta U_{\min } \leq \Delta U_{k} \leq \Delta U_{\max } \\
& U_{\min } \leq U_{k} \leq U_{\max }
\end{aligned}
$$

where $U_{k}$ is an $m \times 1$ input vector, $\left[u_{k}, \ldots, u_{k+m-1}\right]^{T}$.

The resulting problem is a quadratic programming problem with the inequality constraints (36)-(38). Once the optimal inputs $\left[\Delta u_{k}, \ldots, \Delta u_{k+m-1}\right]$ are computed, only the first input $\Delta u_{k}$ is implemented and the rest is discarded. The procedure is repeated at the next sampling time.

In this study, the boiler-turbine system is a MIMO system that has three inputs and three outputs. Therefore, the vectors $Y_{k+1 \mid k}, Y_{k+1 \mid k-1}, Y_{k+1 \mid k}^{d}, R_{k+1 \mid k}$, and $E_{k+1 \mid k}$ are extended to $3 p \times 1$ vectors and $\Delta U_{k}$ is a $3 m \times 1$ vector in (33)-(38). The prediction equation of the boiler-turbine system is then in the following form:

$$
\bar{Y}_{k+1 \mid k}=\bar{Y}_{k+1 \mid k-1}+\bar{S} \Delta \bar{U}_{k}+\bar{Y}_{k+1 \mid k}^{d}
$$

where

$$
\begin{aligned}
& \bar{Y}_{k+1 \mid k}=\left[\begin{array}{llll}
\bar{y}_{k+1 \mid k} & \bar{y}_{k+2 \mid k} & \cdots & \bar{y}_{k+p \mid k}
\end{array}\right]^{T} \\
& =\left[\begin{array}{lll}
\left(y_{1(k+1 \mid k)},\right. & y_{2(k+1 \mid k)} & y_{3(k+1 \mid k)}
\end{array}\right) \\
& \cdots\left(\begin{array}{lll}
y_{1(k+p \mid k)} & y_{2(k+p \mid k)} & y_{3(k+p \mid k)}
\end{array}\right]^{T}
\end{aligned}
$$

$$
\begin{aligned}
\Delta \bar{U}_{k} & =\left[\begin{array}{llll}
\Delta \bar{u}_{k} & \Delta \bar{u}_{k+1} & \cdots & \Delta \bar{u}_{k+m-1}
\end{array}\right]^{T} \\
& =\left[\begin{array}{lll}
\left(\Delta u_{1(k)},\right. & \Delta u_{2(k)}, & \left.\Delta u_{3(k)}\right)
\end{array}\right. \\
& \cdots\left(\begin{array}{lll}
\Delta u_{1(k+m-1)} & \Delta u_{2(k+m-1)} & \left.\Delta u_{3(k+m-1)}\right)
\end{array}\right]^{T} .
\end{aligned}
$$

The subscripts 1-3 in (41) and (43) are the indices for the three outputs and three inputs, and $\bar{S}$ is a $3 p \times 3 m$ dynamic matrix containing nine step responses as follows:

$$
\bar{S}=\left[\begin{array}{cccc}
\bar{s}_{1} & \overline{0} & \cdots & \overline{0} \\
\bar{s}_{2} & \bar{s}_{1} & \ddots & \vdots \\
\vdots & \vdots & \ddots & \bar{s}_{1} \\
\vdots & \vdots & \ddots & \vdots \\
\bar{s}_{p} & \bar{s}_{p-1} & \cdots & \bar{s}_{p-m+1}
\end{array}\right]
$$

where every matrix element $\bar{s}_{i}$ is a $3 \times 3$ vector containing nine amplitudes of the step response at the $i$-th sampling step.

The optimization problem (35) is also extended as follows:

$$
\min _{\Delta \bar{U}_{k}}\left\|\bar{E}_{k+1 \mid k}\right\|_{\Lambda}+\left\|\Delta \bar{U}_{k}\right\|_{\Gamma}
$$

where $\bar{E}_{k+1 \mid k}=\bar{Y}_{k+1 \mid k}-\bar{R}_{k+1 \mid k}$.

$\bar{R}_{k+1 \mid k}$ is fixed with three constant setpoint values. In (45), error and input change are weighted for the three outputs and three inputs as follows:

$$
\begin{gathered}
\left\|\bar{e}_{k+1 \mid k}\right\|=\left[\begin{array}{c}
e_{1(k+1 \mid k)} \\
e_{2(k+1 \mid k)} \\
e_{3(k+1 \mid k)}
\end{array}\right]^{T}\left[\begin{array}{ccc}
1 & 0 & 0 \\
0 & 1 & 0 \\
0 & 0 & 100
\end{array}\right]\left[\begin{array}{l}
e_{1(k+1 \mid k)} \\
e_{2(k+1 \mid k)} \\
e_{3(k+1 \mid k)}
\end{array}\right] \\
\left\|\Delta \bar{u}_{k}\right\|=\left[\begin{array}{c}
\Delta u_{1(k)} \\
\Delta u_{2(k)} \\
\Delta u_{3(k)}
\end{array}\right]^{T}\left[\begin{array}{ccc}
1 & 0 & 0 \\
0 & 1 & 0 \\
0 & 0 & 1
\end{array}\right]\left[\begin{array}{c}
\Delta u_{1(k)} \\
\Delta u_{2(k)} \\
\Delta u_{3(k)}
\end{array}\right]
\end{gathered}
$$

In (46), the weight of the third output error is 100 while other output weights are ones, this is because the nominal value of variables $y_{1}$ and $y_{2}$ are about 100 times than that of $y_{3}$. The three control actions are equally weighted as ones.

$Y_{k+1 \mid k}^{d}$ in (39) is taken as a constant bias of difference between the actual measurement and the open-loop model output. Output constraint (36) is not considered in this study and input constraints (9)-(11) are implemented in the form of (37), and three inputs are constrained in $[0,1]$ in (38).

From theoretical viewpoint, a small sampling time and a large prediction and control horizon are desirable, while they increase the computational burden in practice. Usually, sampling time for discrete control is recommended to be less than $10 \%$ of the smallest time constant. Considering the time constants of $y_{1}$ and $y_{2}$ being about $200 \mathrm{~s}$ from Fig. 3, the sampling time is determined as $5 \mathrm{~s}$. Both prediction and control horizons, $p$ and $m$, are $600 \mathrm{~s}$, in which outputs are settled down as seen in Fig. 3. More extensive analysis to tune the DMC is discussed in [18] and [19].

Fig. 4 shows the system configuration. The DMC controller is applied to the nonlinear boiler-turbine system, and the control algorithm optimizes the control performance (45) at every sampling step with the developed step-response model. For the comparison purpose, the same DMC controller is implemented with both step-response models. 


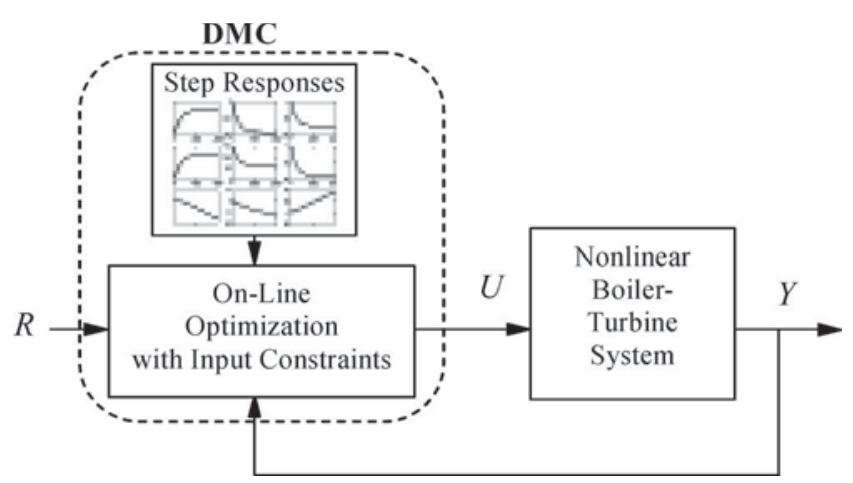

Fig. 4. DMC-based system configuration.

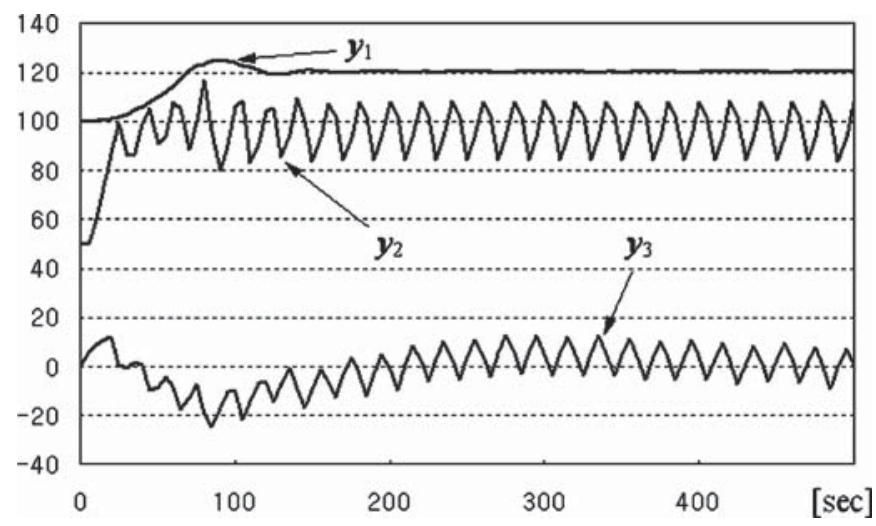

Fig. 5. Responses of the DMC designed with the linearized model.

\section{Simulation Results}

The control system and the process model were simulated with MATLAB in a personal computer environment. The control performance (45) with constraints is optimized with MATLAB function "quadprog ()" at every sampling step.

With the assumption that the system is initially in a steady state $\quad$ with $\quad \bar{y}_{0}=(100,50,0), \bar{x}_{0}=(100,50,449.5), \bar{u}_{0}=$ $(0.271,0.604,0.336)$, several cases are considered. Figs. 5-12 show the simulation results. In the figures, the horizontal axis is time [in seconds], and the vertical axis is [kilograms per centimeter square] for $y_{1}$, [megawatt] for $y_{2}$ and [centimeters] for $y_{3}$ and units for inputs variables are normalized positions of valve actuators for the three inputs $u_{1}, u_{2}$, and $u_{3}$.

In the first case, the DMCs designed with the two stepresponse models in Fig. 3 are compared. In this case, the setpoints of outputs are implemented as $\bar{R}=(120,100,0)$ for both DMCs. This case describes that the setpoints of pressure and electric load are increased to 120 and 100, respectively, while the drum water level is kept to zero.

In Fig. 5, $y_{2}$ and $y_{3}$ show fluctuation, while $y_{1}$ tracks the reference 120 after 150 s. Fig. 6 shows the corresponding control actions. As mentioned in Section II-D, the long-range prediction of $y_{3}$ with linearized model has a limitation for the nonlinear system. Therefore, even if we have a good nonlinear mathematical model of the boiler-turbine system, its linearized model is not suitable for the DMC control of the drum-type boiler-turbine system.

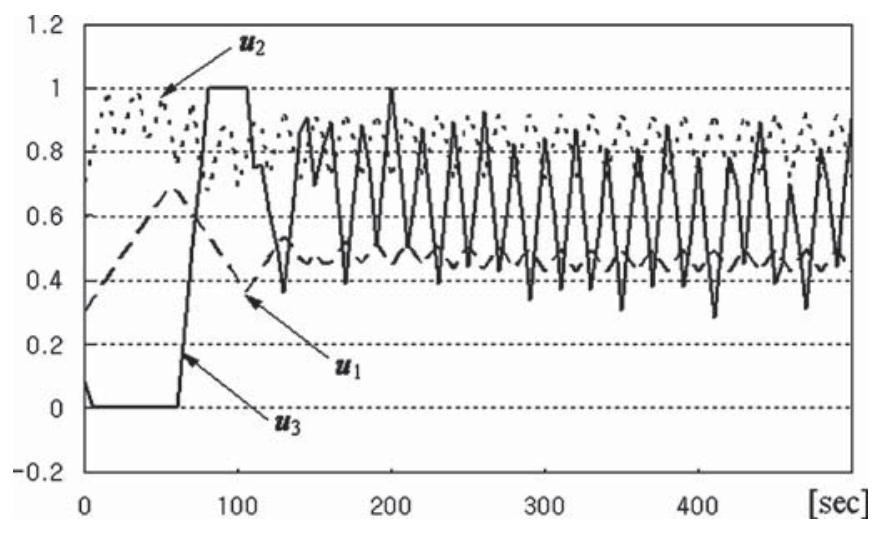

Fig. 6. Control inputs designed with the linearized model.

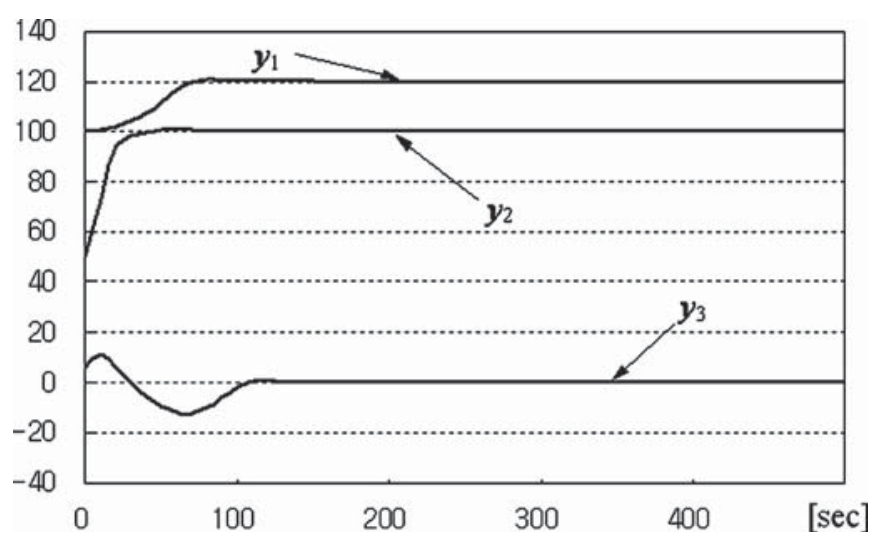

Fig. 7. Responses of the DMC designed with the process test data model.

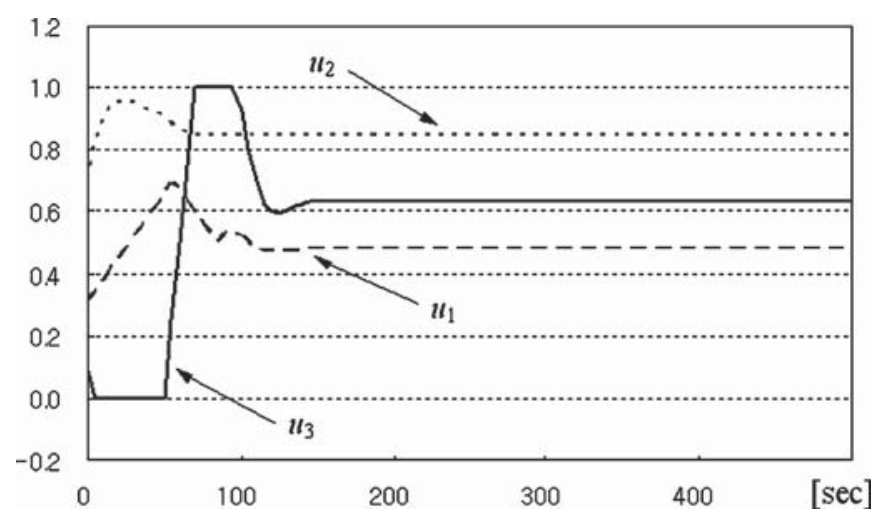

Fig. 8. DMC control inputs designed with the process test data model.

Fig. 7 shows the response of the DMC designed with the process data, where the three outputs are stabilized after $110 \mathrm{~s}$. Fig. 8 shows the corresponding control actions. This result shows that the plant is successfully controlled by the DMC designed with the step-response model obtained by the process data.

To conform the comparison between the linearized model and the process test model, different values of prediction horizon $p$ and control horizon $m$ were tested from 200 to $600 \mathrm{~s}$. And, a different setpoint $\bar{R}$ was tested also. However, similar results, 


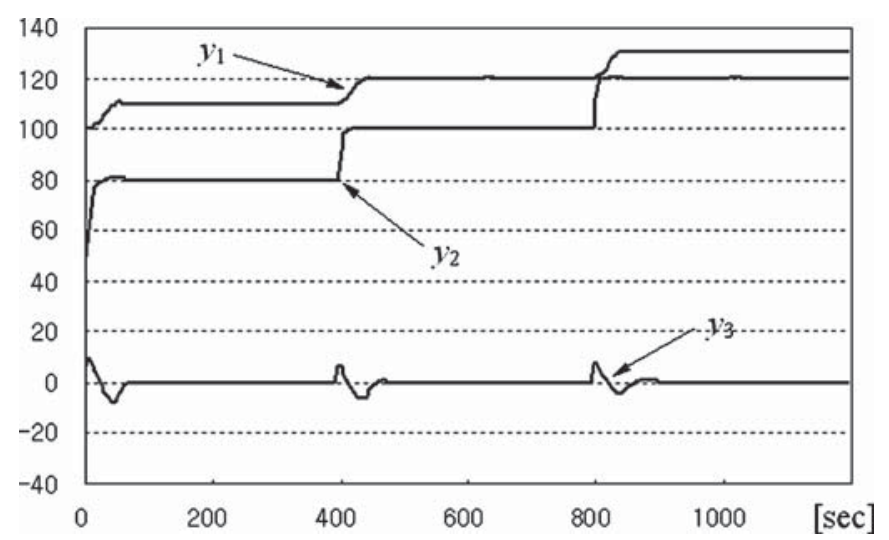

Fig. 9. Response of the DMC designed with the process test data model.

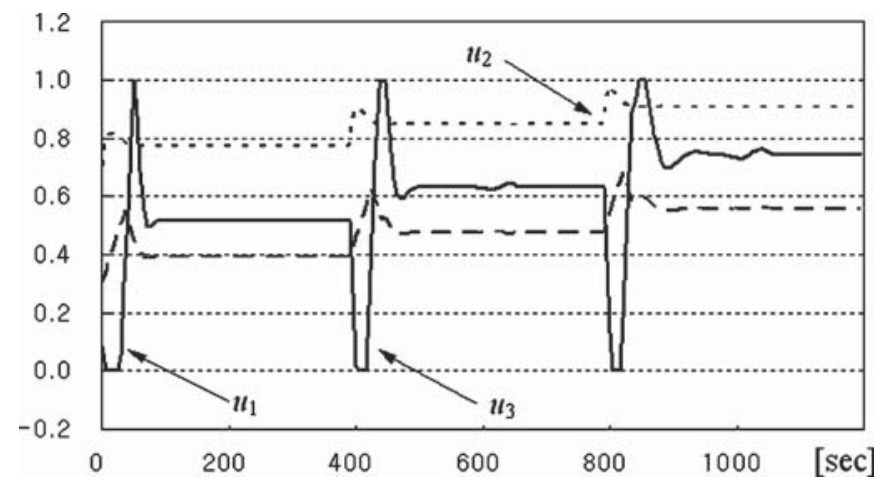

Fig. 10. DMC control inputs designed with the process test data model.

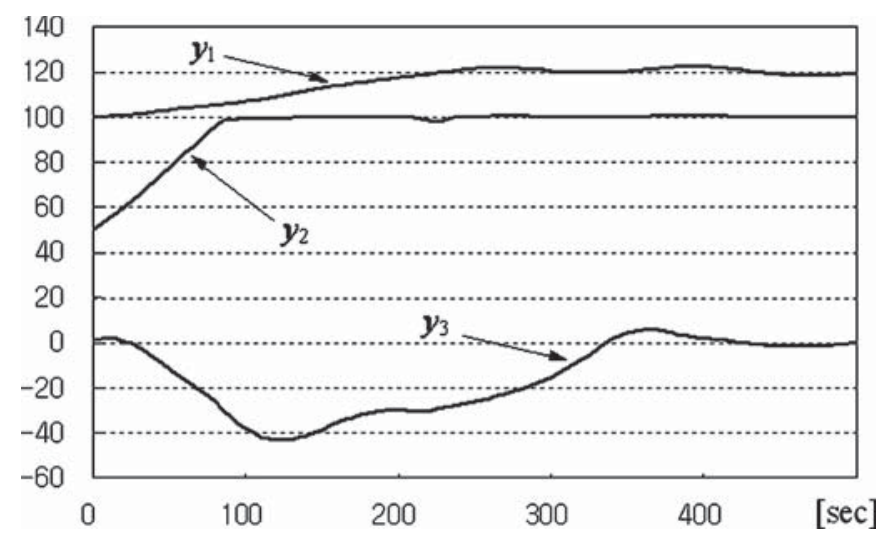

Fig. 11. Process outputs without considering the input constraints.

as shown in Figs. 5-8, were observed in all cases. That is, the process test model is better than linearized model in all cases.

The second case is to demonstrate the tracking ability of DMC. In this case, the setpoints are changed with the DMC designed with the process test data. The setpoint $\bar{R}$ is (110, $80,0)$ initially, and changed to $(120,100,0)$ at 400 and $(130$, $120,0)$ at 800 s, respectively. Figs. 9 and 10, respectively, show the outputs and inputs for this case. Although the step-response model is developed at the operating point, $\bar{y}_{0}=(115,85,0)$, the DMC works well at other operating points, demonstrating its robust characteristics to the boiler-turbine system.

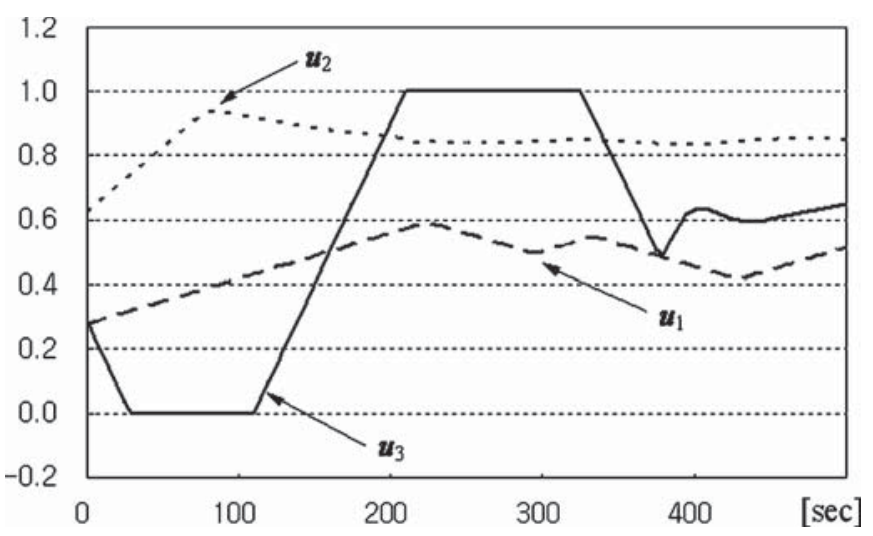

Fig. 12. Process control inputs without considering the inputs constraints.

There are two main reasons about the robustness for the DMC designed with the test data. First, the test data with step inputs have already included the nonlinearity of the boiler-turbine system, while the linearized model is not accurate when the operating point is changed. Second, model mismatch at different operating points is considered as unmeasured disturbance term, $Y_{k+1 \mid k}^{d}$ in (39), which is updated at every sampling step. This eliminates the steady-state error at different operating points.

In the third case, the input constraints, (37) and (38) are removed from the DMC designed with the process test data model. In other words, the inputs are considered only with a weighting factor in (45) in the controller. Therefore, the amplitude and the input change rates are simply chopped with the limit $[0,1]$ and constraints (9)-(11), respectively. In this case, $\bar{R}$ is (120, $100,0)$ as in the first case. Figs. 11 and 12 show the outputs and inputs for this case. In Fig. 11, the settling times of three outputs are increased from those in the Fig. 7. This implies the control performance can be enhanced by considering the input constraints in the DMC algorithm, which is often the case in the real-process industry. This is one of the most important benefits to apply the DMC to the real boiler-turbine system.

\section{CONCLUSION}

This paper presents the application of DMC to a drum-type boiler-turbine system. Two possible step-response models are investigated in designing the DMC; one developed by linearizing the mathematical model and the other developed with the process test data. Because of the high nonlinearity of the drum water-level dynamics, the linearized model is not accurate for relatively large changes in step inputs. On the other hand, the model based on the test data has already reflected the nonlinearity of the model, thus exhibits better responses. Consequently, DMC designed with the process test data model shows satisfactory results while with the linearized model it shows poor results. The DMC shows robust performances for different size of input signals, and prediction and control windows. It also shows good tracking performance and has benefit when considering the input/output constraints, which is often the case in real industrial systems. 
The novel contribution of this paper is as follows: First, it has been shown that the wide-range operation of drum-type boiler-turbine system can be effectively controlled by a direct application of DMC. Until now, the direct application of DMC to drum-type boiler-turbine system was found to be difficult due to the drum-level dynamics with instability and the nonminimum phase. Second, the linearized model of drum-type boiler-turbine system is shown not to be valid for a long-range prediction of DMC, main reason being the poor quality of linearized drum water-level dynamics. Therefore, a careful validation of the stepresponse model is necessary in designing the DMC, although a valid mathematical model is used in this paper. These results can provide a good practical guidance in implementing the DMC.

\section{REFERENCES}

[1] R. D. Bell and K. J. Åström, "Dynamic models for boiler-turbine-alternator units: Data logs and parameter estimation for a $160 \mathrm{MW}$ unit," Lund Inst. Technol., Lund, Sweden, Rep. TFRT-3192, 1987.

[2] B. W. Hogg and N. M. Ei-Rabaie, "Multivariable generalized predictive control of a boiler system," IEEE Trans. Energy Convers., vol. 6, no. 2, pp. 282-288, Jun. 1991.

[3] R. Cori and C. Maffezzoni, "Practical optimal control of a drum boiler power plant," Automatica, vol. 20, pp. 163-173, 1984.

[4] G. Pellegrinetti and J. Bentsman, " $\mathrm{H}_{\infty}$ Controller design for boilers," Int. J. Robust Nonlinear Control, vol. 4, pp. 645-671, 1994.

[5] A. Ben-Abdennour and K. Y. Lee, "A decentralized controller design for a power plant using robust local controllers and functional mapping," IEEE Trans. Energy Convers., vol. 11, no. 2, pp. 394-400, Jun. 1996.

[6] W. Tan, H. J. Marquez, and T. Chen, "Multivariable robust controller design for a boiler system," IEEE Trans. Contr. Syst. Technol., vol. 10, no. 5, pp. 735-742, Sep. 2002.

[7] G. Prasad, E. Swidenbank, and B. W. Hogg, "A neural net model-based multivariable long-range predictive control strategy applied in thermal power plant control," IEEE Trans. Energy Convers., vol. 13, no. 2, pp. 176-182, Mar. 1991.

[8] R. Dimeo and K. Y. Lee, "Boiler-turbine control system design using a genetic algorithm," IEEE Trans. Energy Convers., vol. 10, no. 4, pp. 752759, Dec. 1995.

[9] F. A. Alturki and A. B. Abdennour, "Neuro-fuzzy control of a steam boiler-turbine unit," in Proc. IEEE Int. Conf. Contr. Appl., 1999, pp. 958962.

[10] U.-C. Moon and K. Y. Lee, "A boiler-turbine system control using a fuzzy auto-regressive moving average (FARMA) model," IEEE Trans. Energy Convers., vol. 18, no. 1, pp. 142-148, Mar. 2003.

[11] C. E. Garcia, D. M. Prett, and M. Morari, "Model predictive control: Theory and practice-A survey," Automatica, vol. 25, pp. 335-348, 1989.

[12] J. H. Lee, "Model predictive control in the process industries: Review, current status and future outlook," in Proc. 2nd Asian Contr. Conf, Seoul, Korea, Jul. 22-25, 1997, vol. II, pp. 435-438.

[13] J. Richalet, A. Rault, J. L. Testud, and J. Papon, "Model predictive heuristic control: Application to industrial processes," Automatica, vol. 14, no. 5, pp. 413-428, 1978.

[14] C. R. Culter and B. L. Ramaker, "Dynamic matrix control—A computer control algorithm," presented at the Joint Autom. Contr. Conf., San Francisco, CA, 1980, Paper wp5-b.

[15] J. L. Marchetti, D. A. Mellichamp, and D. E. Seborg, "Predictive control based on discrete convolution models," Ind. Eng. Chem. Proc. Des. Dev., vol. 22 , no. 3, pp. 488-495, 1983.

[16] C. E. Garcia and A. M. Morshedi, "Quadratic programming solution of dynamic matrix control (QDMC)," Chem. Eng. Commun., vol. 46, pp. 7387, 1986.

[17] D. Dougherty and D. Cooper, "A practical multiple model adaptive strategy for multivariable model predictive control," Contr. Eng. Practice, vol. 11, pp. 649-664, 2003.
[18] R. Shridhar and D. J. Cooper, "A tuning strategy for unconstrained multivariable model predictive control," Ind. Eng. Chem. Res., vol. 37, pp. 4003-4016, 1998.

[19] D. Dougherty and D. J. Cooper, "Tuning guidelines of a dynamic matrix controller for integrating (non-self-regulating) processes," Ind. Eng. Chem. Res., vol. 42, pp. 1739-1752, 2003.

[20] J. H. Lee, M. Morari, and C. E. Garcia, "State-space interpretation of model predictive control," Automatica, vol. 30, no. 4, pp. 707-717, 1994.

[21] J. H. Lee and N. L. Ricker, "Extended Kalman filter based model predictive control," Ind. Eng. Chem. Res., vol. 37, pp. 4003-4016, 1998.

[22] Y. Lu and Y. Arkun, "Quasi-min-max MPC algorithm for LPV systems," Automatica, vol. 36, pp. 527-540, 2000.

[23] G. Prasad, G. W. Irwin, E. Swidenbank, and B. W. Hogg, "A hierarchical physical model-based approach to predictive control of a thermal power plant for efficient plant-wide disturbance rejection," Trans. Inst. Meas. Contr., vol. 24, no. 2, pp. 107-128, 2002.

[24] G. Prasad, "On fusion of PCA and a physical model-based predictive control strategy for efficient load-cycling operation of a thermal power plant," Optimal Contr. Appl. Methods, vol. 28, pp. 231-258, 2007.

[25] D. E. Seborg, T. E. Edgar, and D. A. Mellichamp, Process Dynamics and Control. New York: Wiley, 1989.

[26] G. F. Franklin, J. D. Powell, and A. Emami-Naeini, Feedback Control of Dynamic System. Englewood Cliffs, NJ: Prentice-Hall, 2002.

[27] J. A. Rovnak and R. Corlis, "Dynamic matrix based control of fossile power plant," IEEE Trans. Energy Convers., vol. 6, no. 2, pp. 320-326, Jun. 1991.

[28] K. J. Åström and R. D. Bell, "Drum-boiler dynamics," Automatica, vol. 36, pp. 363-378, 2000.

[29] L. A. Sanchez, F. G. Arroyo, and R. A. Villavicencio, "Dynamic matrix control of steam temperature in fossil power plant," in Proc. IFAC Contr. Power Plants Power Syst., Cancun, Mexico, Dec. 1995, pp. 275-280.

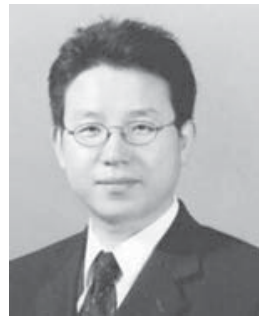

Un-Chul Moon received the B.S., M.S., and Ph.D. degrees from Seoul National University, Seoul, Korea, in 1991, 1993, and 1996, respectively, all in electrical engineering.

From 1996 to 2000, he was with Samsung. In 2000, he joined Woo-Seok University. Since 2002, he has been with Chung-Ang University, Seoul, Korea, where he is currently an Associate Professor of electrical engineering. His current research interests include areas of power system analysis and automations.

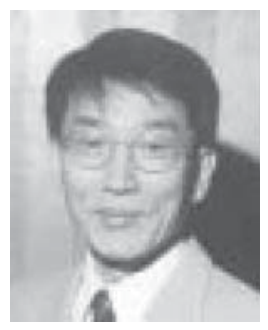

Kwang Y. Lee (S'70-M'72-SM'86-F'01-LF'08) received the B.S. degree in electrical engineering from Seoul National University, Seoul, Korea, in 1964, the M.S. degree in electrical engineering from North Dakota State University, Fargo, in 1968, and the Ph.D. degree in system science from Michigan State University, East Lansing, in 1971.

He was with the faculties of Michigan State, Oregon State, University of Houston, and the Penn State University. Since 2007, he has been with Baylor University, Waco, TX, where he is currently a Professor and the Chair of the Department of Electrical and Computer Engineering. His current research interests include power system control, operation and planning and intelligent system techniques, and their application to power system and power plant control.

Prof. Lee is an Associate Editor of the IEEE TRANSACTIONS ON NEURAL NETWORKS and an Editor of the IEEE TRANSACTIONS ON ENERGY CONVERSION. He is a Registered Professional Engineer. 